\title{
Western diet increases cardiac ceramide content in healthy and hypertrophied hearts
}

Butler, T.J. ${ }^{*}$, Ashford, D. ${ }^{2}$ and Seymour, A-M ${ }^{1}$

${ }^{1}$ School of Biological, Biomedical and Environmental Sciences and Hull York Medical School, University of Hull, Hull, HU6 7RX, UK; ${ }^{2}$ Technology Facility (Proteomics \& Analytical Biochemistry Laboratory) and Centre of Excellence in Mass Spectrometry, Department of Biology, University of York, UK.

*Corresponding Author Dr Thomas Butler, Department of Clinical Sciences and Nutrition, Faculty of Medicine, Dentistry and Clinical Sciences, University of Chester, Parkgate Road, Chester, UK +44 (0) 124451 3090, t.butler@chester.ac.uk.

Short title Impact of western diet on cardiac ceramides

Word count abstract: 248

Word count text: 2994

Number of references: 29

Number of tables: 1

Number of figures: 4

Abbreviations: AC, aortic constriction; CON, control; high-fat diet, HFD; LVH, left ventricular hypertrophy; MCAD, medium-chain acyl-CoA dehydrogenase; SD, standard diet; SPT, serine palmitoyltransferase; SM, sphingomyelin; TAG, triacylglycerol; western diet, WD; 


\begin{abstract}
Background and aims

Obesity and cardiac left ventricular hypertrophy (LVH) are recognised independent risk factors in the development of heart failure (HF). However, the combination of these factors may exacerbate the onset of cardiovascular disease by mechanisms as yet unclear. LVH leads to significant cellular remodelling, including alterations in metabolism which may result in an inappropriate accumulation of lipids and eventual lipotoxicty and apoptosis. The aim of the study was to determine the impact of dietary manipulation on cardiac metabolism in the obese and hypertrophied heart.
\end{abstract}

\title{
Methods and Results
}

LVH was induced via aortic constriction (AC) in an experimental model of cardiac hypertrophy and animals subjected to 9 weeks of dietary manipulation with either a standard, high fat, or a sucrose containing Western-style diet (SD, HFD and WD, respectively). This latter diet resulted in accelerated weight gain in both LVH/AC and control animals. LVH was greater in AC animals fed a WD, and both control and AC animals from this diet showed a significant reduction in cardiac fatty acid oxidation and increased triacylglycerol content. Ceramide content was significantly increased in the WD groups, with no additional effect of LVH. Comparison with a model of HF induced by exposure to Doxorubicin and WD showed exacerbated remodelling of cardiac ceramide species leading to increased C16 and C18 content.

\section{Conclusions}

These findings highlight the inappropriate accumulation and re-distribution of cardiac ceramide species in a diet-induced model of obesity and LVH, potentially increasing susceptibility to cell death. The combination of increased fat and sugar leads to greater pathological remodelling and may explain why this diet pattern is consistently linked with poor cardiovascular outcomes.

\section{Keywords}

cardiac hypertrophy, ceramide, heart failure, high fat diet, $\mathrm{t}$ sugar, western diet, 


\section{Introduction}

Left ventricular hypertrophy (LVH) is a common observation in individuals with obesity, and is positively associated with heart failure (HF) [1,2]. Metabolic remodelling is an early feature of both LVH and HF, with a substantial body of evidence showing that this is characterised by a decline in fatty acid (FA) oxidation (FAO) and increased reliance on glycolytic substrates [3]. Paradoxically, several studies have suggested that supplying fat to the hypertrophied heart prevents the decline in FAO and attenuates the progression of $\mathrm{LVH}[4,5]$. However, more physiological diets such as a Western-diet (WD) - characterised by a moderate amount of fat and sugar - have shown negative effects on cardiac structural and metabolic function in both the healthy or hypertrophied heart [6-11]. These findings suggest that ventricular remodelling is highly susceptible to dietary interventions, and that a modern WD of moderate fat and sugar may have a specific negative effect on the myocardium.

Increased delivery and uptake of FAs - in addition to chronic changes in circulating hormones - leads to accumulation of triacylglycerol (TAG) in the obese heart [12]. The accumulation of TAG is due to divergence of FA uptake and oxidation. Evidence for this derived from observations that greater uptake of FAs via increased expression of the membrane FA translocase, CD36, or reduced mitochondrial uptake and oxidation of FAs, leads to cardiac TAG, diacylglycerol (DAG) and ceramide accumulation, and contributes to a lipotoxic phenotype $[5,13,14]$. The process of lipotoxicity has been recognised as an important pathological mechanism precipitating dysfunction in the obese or hypertrophied heart and is a process that contributes to HF in these conditions [14].

Altered FA handling in both the obese and/or hypertrophied heart and the shunting of activated FAs to ceramide synthesis is an important step in lipotoxicity. Ceramide can be generated either through de novo synthesis requiring serine, palmitoyl-CoA and another fatty acyl-CoA species, or via recycling of more complex sphingolipid species, principally sphingomyelin (SM) [15]. Ceramides of differing chain lengths can be generated from corresponding dihydro-ceramides via the actions of dihydro-ceramide desaturase and also ceramide synthase (CerS) isoforms utilising sphingosine and cytosolic fatty acylCoA species [15] leading to multiple ceramide species in the heart [16, 17]. Indeed, in human patients with severe HF, cardiac ceramides are increased and changes in individual serum ceramide species are observed [18]. Chronic changes to cardiac ceramide species either by diet or LVH may be linked with both HF incidence and progression. The aim of the study was to determine the impact of dietary manipulation on cardiac metabolism in the obese and hypertrophied heart, and whether metabolic variations induce changes in cardiac ceramide species. Additionally, we sought to examine if potential metabolic changes were exacerbated in a model of HF. 
2 Ceramide standards (Cer(d18:1/16:0) [C16], Cer(d18:1/17:0) [C17], $\operatorname{Cer}(\mathrm{d} 18: 1 / 18: 0)$ [C18], $3 \operatorname{Cer}(\mathrm{d} 18: 1 / 20: 0)$ [C20] and $\operatorname{Cer}(\mathrm{d} 18: 1 / 24: 0)$ [C24]) were purchased from Avanti Polar Lipids 4 (Alabaster, MA, USA). Solvents for HPLC were obtained from Fisher Scientific (Loughborough, UK).

5 All other chemicals were purchased from Sigma unless otherwise stated. Male Sprague-Dawley rats 6 (body weight 230-250 g) were obtained from Charles River Laboratories, Kent, UK. Rats had free 7 access to food and water. Experimental protocols were approved by the University ethics committee 8 and conformed to the UK Animals (Scientific Procedures) Act (ASPA) of 1986.

\section{Animal treatment}

Were possible, all procedures followed recommendations for methodological rigor in preclinical cardiovascular studies [19]. Pressure-overload hypertrophy was induced surgically via abdominal aortic constriction (AC) as described previously [10]. Control (CON) animals underwent identical surgery but without AC.

Animals were randomized to one of 3 dietary regimens: $i$ ) standard chow diet (SD, \#801002), ii) high fat diet (HFD, \#824053) or iii) Western Diet (WD, \#824127), giving six experimental groups ( $\mathrm{n}=6$ rats per group): CON-SD, CON-HFD, CON-WD, AC-SD, AC-HFD and AC-WD. Diets were supplied by Special Diet Services (Witham, UK). Sample size was estimated from previous research within our laboratory [10]. Dietary constituents are shown in Table 1. The composition of the HFD and WD was chosen because they are a closer physiological representation of current diet patterns when compared to previous studies in this area, and hence increase relevance of the findings. Rats were maintained on the diets for 9 weeks.

A separate group of animals fed a WD for 9 weeks were treated with Doxorubicin (DOX $n=6$ ) via six intraperitoneal injections of $2.5 \mathrm{mg} / \mathrm{kg}$ over a course of 2 weeks for a total dose of $15 \mathrm{mg} / \mathrm{kg}$ to induce HF [20]. Researchers remained blinded to the intervention (diet and/or AC or DOX) until all results were processed. 
33

Rats were anaesthetised via an intraperitoneal injection of sodium thiopentone $(0.5 \mathrm{ml} / 100 \mathrm{~g})$. Hearts were rapidly excised and perfused briefly to rinse blood from the coronary circulation using a modified isovolumic Langendorff mode with Krebs-Henseleit bicarbonate (KHB) buffer [10] gassed with 95\% $\mathrm{O}_{2} / 5 \% \mathrm{CO}_{2}\left(37^{\circ} \mathrm{C}, \mathrm{pH}\right.$ 7.4).Ventricles were freeze-clamped using liquid nitrogen-cooled Wollenberger tongs and stored at $-80^{\circ} \mathrm{C}$.

\section{Cardiac lipid extraction and analysis}

Frozen cardiac tissue was ground using a liquid nitrogen-cooled pestle and mortar. Total cardiac lipids were extracted from $300 \mathrm{mg}$ powdered ventricular tissue according to the method of Bligh and Dyer [21]. Myocardial TAG were assayed using a Sigma kit (TR0100).

\section{Enzyme activities:}

The activities of medium-chain acyl-CoA dehydrogenase (MCAD) and citrate synthase (CS) were assayed in left-ventricular tissue as described previously [10].

\section{LC/MS detection of cardiac ceramide species}

Cardiac ceramide species were determined in tissue extracts using $300 \mathrm{mg}$ tissue homogenised in PBS ( $\mathrm{pH}$ 7.4). Ceramides were extracted and processed as described by Merrill et al [22]. Chromatography was performed on an Agilent 1100 series pump and autosampler system equipped with an ACE $5 \mu \mathrm{m}$ C18 3x100 mm column (Hichrom Ltd., Berkshire, UK) and a SecurityGuard ${ }^{\mathrm{TM}}$ (Penomenex, UK) guard column with $\mathrm{C}_{18} 4 \times 2 \mathrm{~mm}$ cartridges (Phenomenex). Extracts were resuspended in 50\% methanol. Sample injection volume was $10 \mu \mathrm{l}$. Mobile flow was delivered at a rate of $0.5 \mathrm{ml} / \mathrm{min}$. The HPLC gradient was based on the method of Merrill et al. [22]. The HPLC was controlled using Chromeleon software (Dionex, UK). Electospray ionisation (ESI) TOF-MS spectra were acquired using an API QSTAR Pulsar i tandem mass spectrometer (AB Sciex Instruments) in the positive ion mode using a TurbolonSpray source. Standard curves for C16, C18, C20 and C24 ceramides relative to the C17 ceramide internal standard were prepared and used to determine the concentration of analytes in cardiac extracts. 


\section{Statistical analyses}

63 Data are presented as mean \pm SE. Multiple comparisons were performed by using a one-way ANOVA 64 with post hoc Sheffé test. Data were analysed using IBM SPSS statistics (v. 23.0) with a statistical 65 significant result defined as a $P$ value $<0.05$. 


\section{Results}

\section{Cardiac morphological and metabolic changes are exacerbated by a western diet}

CON-SD and AC-SD showed a $111 \%$ and 115\% increase in body weight over the 9 week period, respectively. In contrast, CON-HFD and AC-HFD animals demonstrated a 136\% and 119\% increase in body weight over the 9 week period, respectively. Similar increases body weight were observed for the WD group (174\% and 140\%, CON and AC respectively) (Fig. 1). CON animals fed WD displayed a significant increase in terminal body weight in comparison to the CON-SD and CON-HFD groups. No significant difference were observed for AC groups. No significant differences in tibia length (TL) were observed between groups, suggesting that differences in body weight were not due to increases in lean body mass (results not shown).

Heart weight was increased by AC in all dietary groups when compared to their respective controls. However, this increase only reached significance in SD and WD groups $(P<0.05)$. Mean heart weight was $2.01 \pm 0.1,2.60 \pm 0.1,2.16 \pm 0.1,2.41 \pm 0.2,2.18 \pm 0.2$ and $2.65 \pm 0.2 \mathrm{~g}$ in CON-SD, AC-SD, CON-HFD, AC-HFD, CON-WD and AC-WD groups, respectively). Heart weight did not differ significantly between control groups. When heart weight was normalised to TL as a marker of LVH [23] all AC groups showed a significant increase in the degree of hypertrophy when compared to their respective controls although this was more pronounced in the AC-WD group (Fig. 2A).

No significant differences were found in MCAD activity between groups. Mean MCAD activity was $12.0 \pm 1.0,11.0 \pm 1.0,13.4 \pm 0.6,12.1 \pm 0.5,13.8 \pm 0.3$ and $12.0 \pm 0.4 \mu \mathrm{mol} / \mathrm{min} / \mathrm{g}$ in CON-SD, ACSD, CON-HFD, AC-HFD, CON-WD and AC-WD groups, respectively. The HFD significantly increased CS activity in both CON and AC hearts when compared to the SD $(73.4 \pm 3.1$ and $65.1 \pm 2.4$ vs. $55.2 \pm 2.9,46.1 \pm 1.8 \mu \mathrm{mol} / \mathrm{min} / \mathrm{g}$, respectively, $P<0.05)$. WD feeding led to a significant increase $(P<0.05)$ in CS activity in both CON and AC groups $(101.4 \pm 2.2,96.2 \pm 3.5 \mu \mathrm{mol} / \mathrm{min} / \mathrm{g}$, respectively $)$ when compared to SD and HFD groups. These results in both the HFD and WD groups are suggestive of a specific mitochondrial biogenic response following these dietary feeding patterns. No differences in $\mathrm{CS}$ activity were observed between $\mathrm{CON}$ and $\mathrm{AC}$ groups within the same dietary regimen. The increase in CS activity coupled with unaltered MCAD activity suggested a decrease in the capacity for $\beta$-oxidation in WD hearts, shown by a reduced MCAD:CS ratio [24] (Fig. 2B). 


\section{Myocardial triacylglycerol content is elevated in hearts from western diet-fed rats}

Cardiac TAG was significantly increased $(P<0.01)$ by WD feeding in both CON and AC groups $(2.4 \pm$ 0.1 and $2.3 \pm 0.3 \mu \mathrm{mol} / \mathrm{g}$, respectively, ) when compared to their SD and HFD counterparts (1.6 \pm 0.1 , $1.7 \pm 0.1,1.6 \pm 0.1$ and. $1.5 \pm 0.2 \mu \mathrm{mol} / \mathrm{g}$, respectively). No intra-group differences were observed.

\section{Altered long-chain ceramide content in Western diet hearts}

No significant difference between any of the ceramide species measured in animals fed either a SD or HFD, or those subjected to AC (Fig. 3A-D). WD was associated with a substantial increase in all ceramides assayed, with no discernible difference between CON and AC groups (Fig. 3A-D). Irrespective of dietary intervention or AC, the predominant ceramide species measured was C24 (Fig. 3D).

\section{Doxorubicin treatment specifically alters the cardiac lipid and ceramide profile associated with a western diet}

Administration of DOX led to deterioration in health including lethargy, poor coat condition and porphyrin staining around the nose and eyes, culminating in a $17 \%$ mortality.

Cardiac TAG content was increased following DOX when compared to the CON group however this did not reach statistical significance ( $3.1 \pm 0.6$ vs. $2.3 \pm 0.2 \mu \mathrm{mol} / \mathrm{g}$, respectively, $P=0.06$ ). This is likely due to a reduction in statistical power due to $17 \%$ mortality. DOX treatment significantly increased total cardiac ceramide content when compared to the CON group, driven by significant increases in C16 and C18 ceramide (Fig. 4A-B). Concentrations of these ceramide species were increased 2.7- and 3.4-fold following DOX treatment, respectively. C20 and C24 ceramide were unaltered by DOX treatment (Fig. 4C-D). Overall, DOX administration led to the greatest increase in cardiac ceramide (Fig. 4E). 


\section{Discussion}

The present study demonstrated that a WD with modest amounts of fat and sugar can cause a substantial increase in the HW in animals subjected to AC and increases TAG content of both CON and hypertrophied hearts. The metabolic alterations of reduced capacity for FAO coupled with increased TAG are associated with a significant increase in cardiac ceramide content which may render the heart more susceptible to apoptosis, cell loss and ultimately HF.

Neither HW nor the HW:TL was altered by dietary intervention alone which is in contrast to recent work examining the effect of similar dietary interventions. Neves et al. [11] showed that left ventricular weight was increased by $12.5 \%$ in Swiss mice subjected to 16 weeks of similar dietary intervention, and Tikellis et al. [9] have observed myocyte hypertrophy following 16 weeks of a WD. The discrepancy between our data and previous work likely reflects differences in duration of study, age of animals used, the magnitude of sucrose or fructose consumption and source of dietary fat. Of particular interest in our study was the observed greater degree of hypertrophy in the AC-WD group. These observations suggest that WD combined with the stimulus for ventricular growth leads to a more pronounced hypertrophy. This is further supported by the blunted hypertrophic response seen in ACHFD animals. Several mechanisms have been postulated to support this, including an important role of elevated plasma insulin and increased insulin signalling leading in accelerated protein synthesis and greater remodelling in animals fed a high carbohydrate diet [14]. We believe that a strength of our approach is the \%kcal contributions from different macronutrients, which reinforces the relevance of the findings. In particular we observed that an increase from $9 \%$ to $14 \%$ of total calories from sucrose is sufficient to precipitate greater structural and metabolic remodelling.

We also observed enzymatic changes which were largely dependent on the dietary intervention. Mitochondrial biogenesis was observed following both the HFD and WD in CON and AC animals (shown by an increase in CS activity) in comparison to SD counterparts. Mitochondrial transcription factor A (TFAM) has been shown to be increased in the hearts of animals fed a WD and may be due to oxidative stress generated from this dietary pattern $[6,8]$. However, despite a biogenic response FAO in the WD-fed animals was reduced as measured by the MCAD:CS ratio, and was positively associated with TAG accumulation. The greatest reduction in FAO was found in the AC-WD group, consistent with others $[3,11]$. The mechanism behind such a reduction remains unclear however inadequate activation of the transcription factor peroxisome proliferator-a $c t$ i $v$ a t e d $r$ e c e p t o $r$ previously reported following WD [8] and thus may be the cause of the reduced FAO observed in both CON-WD and AC-WD groups. Our data clearly highlight the detrimental impact of increasing sucrose 
intake in combination with a moderate amount of fat. It would be prudent to examine the impact of the same percentage of dietary fat in combination with $0 \%$ sucrose on ventricular remodelling.

Cardiac ceramide species were elevated in hearts from WD-fed animals. Mechanistically, the origin of the increase in cardiac ceramide species in our study remains undetermined. Alterations to cardiac acylCoA species and therefore alterations to the cardiac acyl-CoA pool following the WD [7] could lead to increased shunting of these species into the de novo pathway of ceramide formation. This is supported by the observation that a WD decreases the conversion of palmitate to palmitoleic acid in the myocardium and reduces the ratio of MUFA:SFA, creating a palmitate-rich environment that favours ceramide formation [7]. We observed C24 ceramide to be the most predominant species in our platform of ceramides analysed, consistent with previous observations [16, 17]. Importantly, these studies demonstrate that ceramide remodelling extends further than the traditionally studied C16 ceramide and exposes the sole measurement of this sphingolipid as being of limited use.

We further sought to set our results in the context of cardiac remodelling by utilising the DOX model of HF [20]. In this model we observed a specific increase in C16 and C18 ceramide. The rise in C16 ceramide may be explained by the increase in palmitate observable following WD feeding [7]. The origins of the substantial increase in $\mathrm{C} 18$ ceramide are likely complex. It appears that the cardiotoxicity related to DOX is exacerbated in animals fed a HFD, mediated through increased oxidative stress and uncoupling of ATP provision, and decreased FAO [25]. Earlier studies have shown that a WD increases stearoyl-CoA [7] and DOX has been shown to activate ceramide synthase 1 (Cers1, responsible for the synthesis of $\mathrm{C} 18$ ceramide) [26]. Furthermore, the authors showed that this activation was associated with increased expression of p38 MAP kinase with the potential for enhanced apoptosis which could lead to impaired cardiac function. Combined with an increase in stearoyl-CoA following a WD, decreased FAO from DOX treatment and increased expression of CerS1 may explain the specific accumulation of $\mathrm{C} 18$ ceramide in the present DOX model.

\section{Strengths and limitations}

The benefits of the abdominal AC model of pressure overload model of LVH have been reviewed extensively [27], ultimately requiring a less severe surgical procedure and displaying a reduced mortality in comparison to more severe models of ventricular hypertrophy. Our laboratory has previously shown that this is a compensated cardiac phenotype useful for examining early changes in metabolism [10]. In comparison, transverse aortic constriction induces a much more severe cardiac 
response making it unsuitable for studying the early metabolic and physiological changes that occur during ventricular remodelling. Furthermore, because the rats used in the present study were 8 weeks of age at the time of the procedure, the abdominal banding leads to a gradual increase in afterload, mimicking progressive hypertension and increasing the translational nature of the findings.

We chose to use a diet-induced model of obesity in order to maximise the translational impact of the findings. Indeed, common models such as $o b / o b$ and $d b / d b$ mice, in addition to the $f a / f a$ rat which utilise disrupted leptin signalling to induce hyperphagia and obesity - also can impact upon cardiac structure independently of body weight [28]. The strength of our approach was to utilise a rodent model where weight-gain was induced purely by diet, without any genetic manipulation. The composition of the HFD and WD was chosen because they are a closer physiological representation of current diet patterns and hence increase relevance of the findings.

There are several limitations to our work that warrant discussion. We did not investigate changes in feeding behaviour between the different groups, however previous studies have shown a WD to be highly palatable [29]. Secondly, we utilised only male animals and as such, these experiments should be repeated in female rodents. Thirdly, our results should be interpreted within the context of early ventricular remodelling, and may not represent changes in more severe LVH and HF. However, this may be regarded as a strength, as we show that even in an early compensated model, pathological alterations are observable which may precede the development of more severe structural and metabolic changes. Further studies are required to investigate if our observations translate into worse remodelling over a longer study duration.

\section{Conclusions}

Our data provide evidence that the WD is capable of inducing substantial metabolic remodelling in the heart when compared to both the SD and HFD, and is consistent with the concept of glucolipotoxicity. The WD led to the greatest weight gain, characterised by higher fat mass and central adiposity mimicking obesity. The novelty of our work highlights that changes in cardiac ceramide species are strongly influenced by diet and less-so by cardiac hypertrophy at this early stage of remodelling. Over time, chronic changes in ceramide may contribute to the transition from compensated hypertrophy to end-stage HF [30]. 


\section{Acknowledgments}

TJB was supported by a PhD studentship from University of Hull Clinical Biosciences Institute and a grant from The Hull and East Riding Cardiac Research Trust Fund. The funding bodies had no involvement with the study design, collection of data, analysis and preparation of the manuscript. 
[1] Avelar E, Cloward TV, Walker JM, Farney RJ, Strong M, Pendleton RC, et al. Left ventricular hypertrophy in severe obesity: interactions among blood pressure, nocturnal hypoxemia, and body mass. Hypertension. 2007;49:34-9.

[2] Crump C, Sundquist J, Winkleby MA, Sundquist K. Aerobic fitness, muscular strength and obesity in relation to risk of heart failure. Heart. 2017.

[3] Lai L, Leone TC, Keller MP, Martin OJ, Broman AT, Nigro J, et al. Energy Metabolic Reprogramming in the Hypertrophied and Early Stage Failing Heart: A Multisystems Approach. Circulation: Heart Failure. 2014;7:1022-31.

[4] Okere IC, Chess DJ, McElfresh TA, Johnson J, Rennison J, Ernsberger P, et al. High-fat diet prevents cardiac hypertrophy and improves contractile function in the hypertensive dahl salt-sensitive rat. Clin Exp Pharmacol Physiol. 2005;32:825-31.

[5] Okere IC, Young ME, McElfresh TA, Chess DJ, Sharov VG, Sabbah HN, et al. Low

carbohydrate/high-fat diet attenuates cardiac hypertrophy, remodeling, and altered gene expression in hypertension. Hypertension. 2006;48:1116-23.

[6] Ballal K, Wilson C, Harmancey R, Taegtmeyer H. Obesogenic high fat western diet induces oxidative stress and apoptosis in rat heart. Molecular and Cellular Biochemistry. 2010;344:221-30.

[7] Harmancey R, Wilson CR, Wright NR, Taegtmeyer H. Western diet changes cardiac acyl-CoA composition in obese rats: a potential role for hepatic lipogenesis. Journal of Lipid Research.

2010;51:1380-93.

[8] Wilson CR, Tran MK, Salazar KL, Young ME, Taegtmeyer H. Western diet, but not high fat diet, causes derangements of fatty acid metabolism and contractile dysfunction in the heart of Wistar rats.

Biochem J. 2007;406:457-67.

[9] Tikellis C, Thomas MC, Harcourt BE, Coughlan MT, Pete J, Bialkowski K, et al. Cardiac

inflammation associated with a Western diet is mediated via activation of RAGE by AGEs. Am J Physiol Endocrinol Metab. 2008;295:E323-30.

[10] Akki A, Seymour AM. Western diet impairs metabolic remodelling and contractile efficiency in cardiac hypertrophy. Cardiovasc Res. 2009;81:610-7.

[11] Neves FA, Cortez E, Bernardo AF, Mattos AB, Vieira AK, Malafaia Tde O, et al. Heart energy metabolism impairment in Western-diet induced obese mice. J Nutr Biochem. 2014;25:50-7.

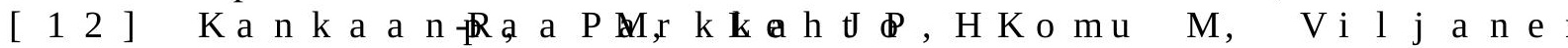
triglyceride content and epicardial fat mass in human obesity: relationship to left ventricular function and serum free fatty acid levels. The Journal of Clinical Endocrinology \& Metabolism. 2006;91:468995.

[13] Ouwens D, Diamant M, Fodor M, Habets D, Pelsers M, El Hasnaoui M, et al. Cardiac contractile dysfunction in insulin-resistant rats fed a high-fat diet is associated with elevated CD36-mediated fatty acid uptake and esterification. Diabetologia. 2007;50:1938-48.

[14] Stanley WC, Dabkowski ER, Ribeiro RF, O'Connell KA. Dietary Fat and Heart Failure: Moving From Lipotoxicity to Lipoprotection. Circulation Research. 2012;110:764-76.

[15] Hannun YA, Obeid LM. Principles of bioactive lipid signalling: lessons from sphingolipids. Nat Rev Mol Cell Biol. 2008;9:139-50.

[16] Monette JS, Gómez LA, Moreau RF, Bemer BA, Taylor AW, Hagen TM. Characteristics of the rat cardiac sphingolipid pool in two mitochondrial subpopulations. Biochemical and biophysical research communications. 2010;398:272-7.

[17] Baranowski M, Blachnio A, Zabielski P, Gorski J. PPARalpha agonist induces the accumulation of ceramide in the heart of rats fed high-fat diet. Journal of Physiology and Pharmacology.

2007;58:57.

[18] Ji R, Akashi H, Drosatos K, Liao X, Jiang H, Kennel PJ, et al. Increased de novo ceramide synthesis and accumulation in failing myocardium. JCI Insight. 2017;2:e82922.

[19] Ramirez FD, Motazedian P, Jung RG, Di Santo P, MacDonald ZD, Moreland R, et al.

Methodological Rigor in Preclinical Cardiovascular Studies: Targets to Enhance Reproducibility and Promote Research Translation. Circulation Research. 2017. 
[20] Lu L, Wu W, Yan J, Li X, Yu H, Yu X. Adriamycin-induced autophagic cardiomyocyte death plays a pathogenic role in a rat model of heart failure. International Journal of Cardiology. 2009;134:82-90.

[21] Bligh EG, Dyer WJ. A rapid method of total lipid extraction and purification. Canadian journal of biochemistry and physiology. 1959;37:911-7.

[22] Merrill AH, Jr., Sullards MC, Allegood JC, Kelly S, Wang E. Sphingolipidomics: highthroughput, structure-specific, and quantitative analysis of sphingolipids by liquid chromatography tandem mass spectrometry. Methods. 2005;36:207-24.

[23] Yin F, Spurgeon HA, Rakusan K, Weisfeldt ML, Lakatta EG. Use of tibial length to quantify cardiac hypertrophy: application in the aging rat. American Journal of Physiology-Heart and Circulatory Physiology. 1982;243:H941-H7.

[24] Chess DJ, Lei B, Hoit BD, Azimzadeh AM, Stanley WC. Deleterious effects of sugar and protective effects of starch on cardiac remodeling, contractile dysfunction, and mortality in response to pressure overload. American Journal of Physiology - Heart and Circulatory Physiology. 2007;293:H1853-H60.

[25] Mitra MS, Donthamsetty S, White B, Mehendale HM. High fat diet-fed obese rats are highly sensitive to doxorubicin-induced cardiotoxicity. Toxicology and applied pharmacology.

2008;231:413-22.

[26] Min J, Mesika A, Sivaguru M, Van Veldhoven PP, Alexander H, Futerman AH, et al.

(Dihydro)ceramide Synthase 1-Regulated Sensitivity to Cisplatin Is Associated with the Activation of p38 Mitogen-Activated Protein Kinase and Is Abrogated by Sphingosine Kinase 1. Molecular Cancer Research. 2007;5:801-12.

[27] Berry JM, Naseem RH, Rothermel BA, Hill JA. Models of cardiac hypertrophy and transition to heart failure. Drug Discovery Today: Disease Models. 2007;4:197-206.

[28] Hall ME, Harmancey R, Stec DE. Lean heart: Role of leptin in cardiac hypertrophy and metabolism. World Journal of Cardiology. 2015;7:511-24.

[29] La Fleur S, Luijendijk M, Van Der Zwaal E, Brans M, Adan R. The snacking rat as model of human obesity: effects of a free-choice high-fat high-sugar diet on meal patterns. International Journal of Obesity. 2014;38:643.

[30] Ji R, Akashi H, Drosatos K, Liao X, Jiang H, Kennel PJ, et al. Increased de novo ceramide synthesis and accumulation in failing myocardium. JCI Insight. 2017;2:e82922. 
Figure 1

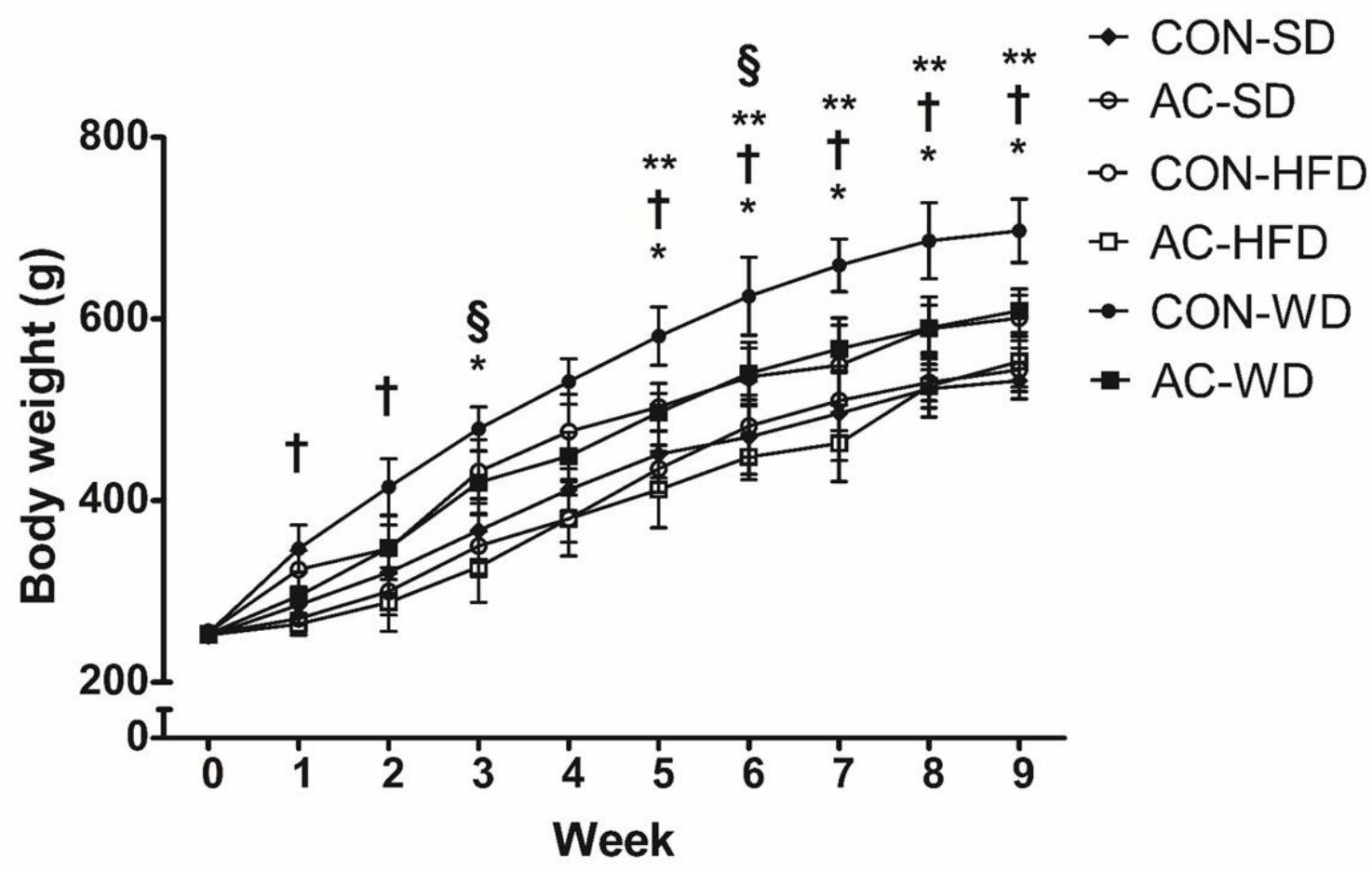

Fig. 1. Western diet feeding leads to significant body weight gain. Weekly body weights were assessed in male Sprague-Dawley rats after either aortic constriction (AC) or a control (CON) procedure and a standard, high-fat or western diet (SD, HFD and WD, respectively) for 9 weeks. Data are means \pm SE of $\mathrm{n}=6$ animals per group. $*=P<0.05$ CON-HFD vs. AC-H F D ; $P \varangle 0.05 € O O N-$ WD vs. AC-WD; $* *=P<0.05$ CON-WD vs. CON-SD and CON-HFD; $\S=P<0.05$ AC-WD vs. ACSD and AC-HFD. 


\section{Figure 2}

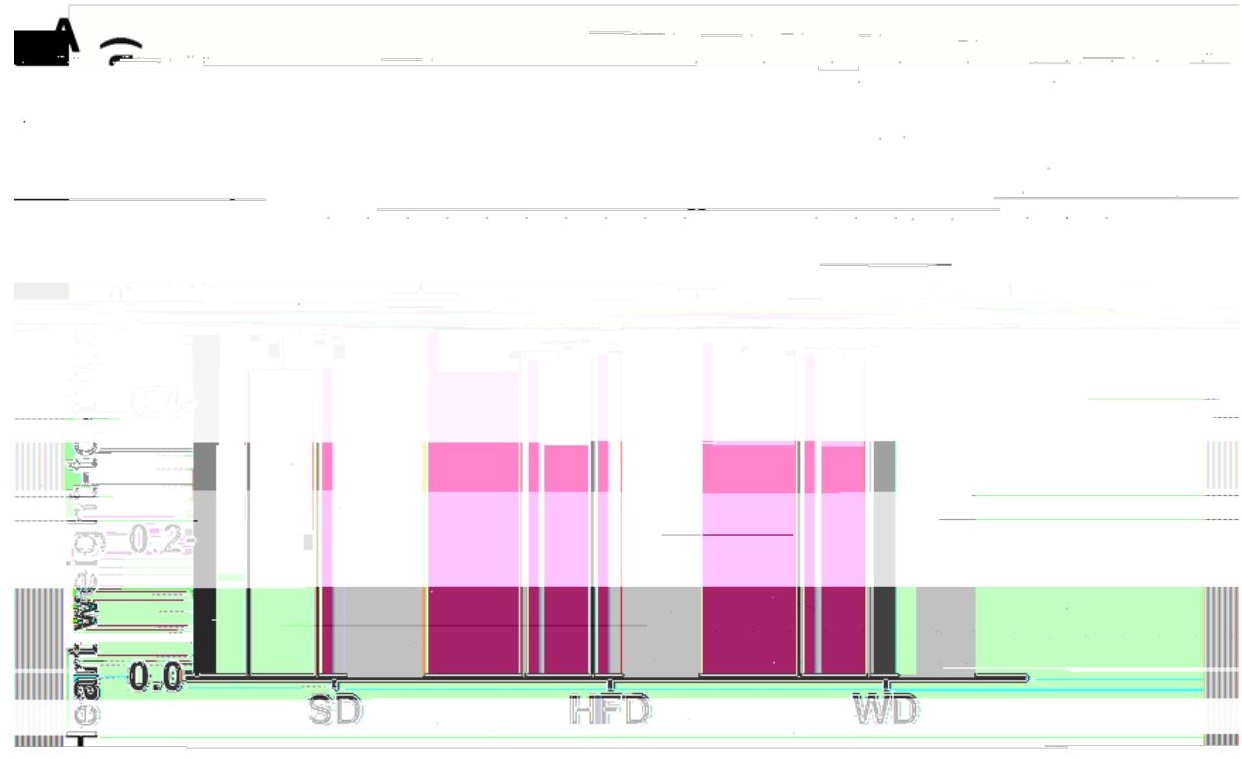

(69.

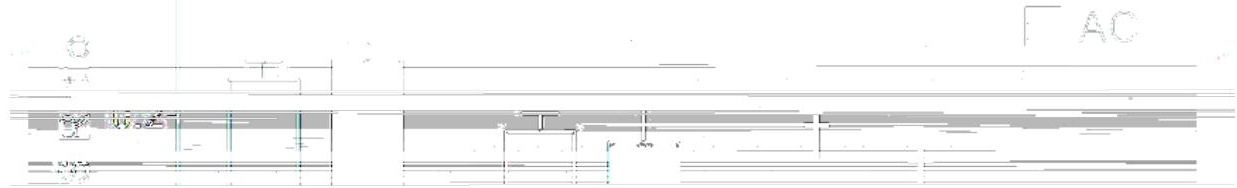

Fig. 2. Western diet regulates the cardiac response to aortic constriction and is associated with decreased capacity for fatty acid oxidation. A) Heart weight-to-tibia length and B) MCAD:CS ratios were determined in male Sprague-Dawley rats after either aortic constriction (AC, filled bars) or a control (CON, open bars) procedure and a standard, high-fat or western diet (SD, HFD and WD, respectively) for 9 weeks. Data are means \pm SE of $n=6$ animals for SD groups, $n=6$ for HFD groups, and $\mathrm{n}=11$ for CON-WD and $\mathrm{n}=6$ for AC-WD. $*=P<0.05$ vs. respective control; $* *=$

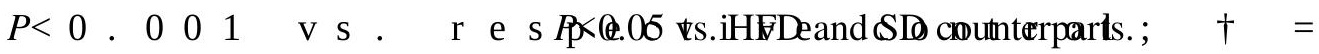




\section{Figure 4}

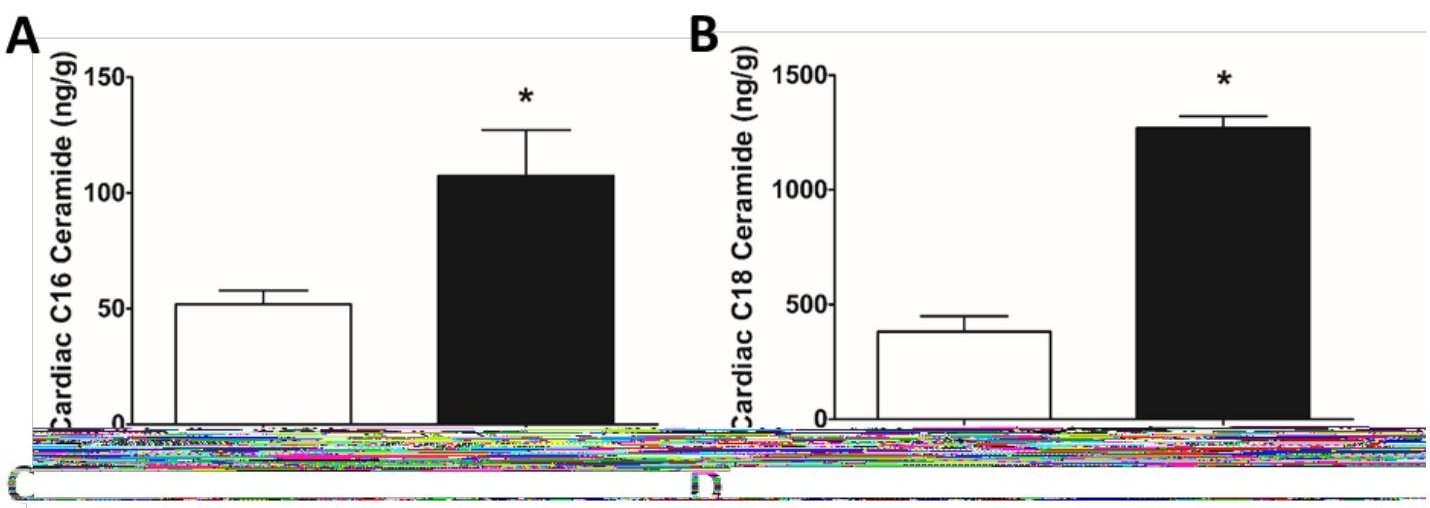

Fig. 4. Doxorubicin (DOX) administration to animals fed a Western diet leads to specific alteration in the cardiac long-chain ceramide pool. Myocardial content of C16 (A), C18 (B), C20 (C) and C24 (D) ceramide was analysed in cardiac extracts prepared from the hearts of male Sprague-Dawley rats after either doxorubicin (DOX, filled bars) or saline administration control (CON, open bars) and a western diet (WD) for 9 weeks. DOX administration led to the greatest increase in ceramide levels (E). Data are means \pm SE of $n=6$ for HFD groups, $n=11$ for CON-WD, $n=6$ for AC-WD and $n=5$ for DOXWD. $*=P<0.01$ vs. CON-WD; $+=P<0.001$ vs. HFD counterparts; $\#=P<0.05$ vs. SD, HFD, CONWD, and AC-WD. 
Tables

Table 1 Dietary macronutrient composition

Diet

\begin{tabular}{|c|c|c|c|c|c|c|}
\hline \multirow[b]{2}{*}{ Dietary Component } & \multicolumn{2}{|c|}{$\mathrm{SD} 3.3 \mathrm{kcal} / \mathrm{g}$} & \multicolumn{2}{|c|}{ HFD $4.54 \mathrm{kcal} / \mathrm{g}$} & \multicolumn{2}{|c|}{ WD $4.56 \mathrm{kcal} / \mathrm{g}$} \\
\hline & $\%(\mathrm{w} / \mathrm{w})$ & $\begin{array}{c}\text { Energy } \\
(\%)\end{array}$ & $\%(\mathrm{w} / \mathrm{w})$ & $\begin{array}{c}\text { Energy } \\
(\%)\end{array}$ & $\%(\mathrm{w} / \mathrm{w})$ & $\begin{array}{c}\text { Energy } \\
(\%)\end{array}$ \\
\hline Carbohydrate (Total) & 61.7 & 74.8 & 39.8 & 35.1 & 40.3 & 35.0 \\
\hline Sucrose & 4.1 & 5.0 & 10.5 & 9.3 & 16.0 & 13.9 \\
\hline Protein (Casein) & 14.4 & 17.5 & 23.0 & 20.0 & 23.0 & 20.0 \\
\hline Fats (Total Lard + Soya & & & & & & \\
\hline Oil) & 2.7 & 7.4 & 22.6 & 45.0 & 22.5 & 45.0 \\
\hline Fibre & 4.7 & - & 4.6 & - & 4.6 & - \\
\hline
\end{tabular}

SD, standard diet; HFD, high-fat diet; WD, western diet. 
Tables

Table 1 Dietary macronutrient composition

Diet

\begin{tabular}{|c|c|c|c|c|c|c|}
\hline \multirow[b]{2}{*}{ Dietary Component } & \multicolumn{2}{|c|}{$\mathrm{SD} 3.3 \mathrm{kcal} / \mathrm{g}$} & \multicolumn{2}{|c|}{ HFD $4.54 \mathrm{kcal} / \mathrm{g}$} & \multicolumn{2}{|c|}{ WD $4.56 \mathrm{kcal} / \mathrm{g}$} \\
\hline & $\%(\mathrm{w} / \mathrm{w})$ & $\begin{array}{c}\text { Energy } \\
(\%)\end{array}$ & $\%(\mathrm{w} / \mathrm{w})$ & $\begin{array}{c}\text { Energy } \\
(\%)\end{array}$ & $\%(\mathrm{w} / \mathrm{w})$ & $\begin{array}{c}\text { Energy } \\
(\%)\end{array}$ \\
\hline Carbohydrate (Total) & 61.7 & 74.8 & 39.8 & 35.1 & 40.3 & 35.0 \\
\hline Sucrose & 4.1 & 5.0 & 10.5 & 9.3 & 16.0 & 13.9 \\
\hline Protein (Casein) & 14.4 & 17.5 & 23.0 & 20.0 & 23.0 & 20.0 \\
\hline Fats (Total Lard + Soya & & & & & & \\
\hline Oil) & 2.7 & 7.4 & 22.6 & 45.0 & 22.5 & 45.0 \\
\hline Fibre & 4.7 & - & 4.6 & - & 4.6 & - \\
\hline
\end{tabular}

SD, standard diet; HFD, high-fat diet; WD, western diet. 